\title{
Potential role of medicinal plants against Alzheimer's disease
}

\begin{abstract}
Alzheimer's disease (AD) is a progressive neurodegenerative disorder of the brain which leads to a decline in memory and cognition. The Food and Drug Administration (FDA) approved medications provide only symptomatic relief and are often associated with adverse effects. Many natural products have shown their activities against AD. Bioactive compounds like polyphenols, tannins, alkaloids, flavonoids, terpenes, sterols, etc are present in various plants. These compounds have shown antioxidant, anti-inflammatory, anticholinesterase, and anti-amyloidogenic activities. This review article discusses the benefits of various plants against AD.
\end{abstract}

Volume 14 Issue 3 - 202 I

\author{
Supriya Yadav \\ Department of Siddha Medicine, Eastern University, India
}

Correspondence: Supriya Yadav, Department of Siddha Medicine, Eastern University, India, Tel 9167498104,

Email supriyayadav0219@gmail.com

Received: February 04, 202I | Published: May 27, 2021

Keywords: alzheimer's disease, food and drug administration, glutamate excitotoxicity, cholinergic system

Abbreviations: AD, Alzheimer's disease; FDA, food and drug administration; GPX, glutathione peroxidase; MO, moringa olifera; LPO, lipid peroxides; OGD, oxygen glucose deprivation

\section{Introduction}

Alzheimer's disease (AD) is a type of chronic neurodegenerative disease. It has many causes: accumulation of amyloid beta and neurofibrillary tangles, epigenetic changes, glutamate excitotoxicity, cholinergic system dysfunction, oxidative stress, and inflammation. ${ }^{1,2}$ $\mathrm{AD}$ leads to irreversible loss of synapses and neurons. Therefore, the two hallmarks are plaques of amyloid beta and neurofibrillary tangles. Hippocampus is the first site where the damage appears initially. Hippocampus is a crucial part of the brain, which forms memories. As time passes, more neurons die and the brain begins to shrink. In the final stage, the brain tissue has shrunk remarkably. It is responsible for about $60 \%$ of dementia cases. Every 1 in 8 people over the age of 65 while, nearly half over the age of 85 have AD. ${ }^{3}$

The initial stage of the disease shows changes in the brain but no symptoms. The mild cognitive impairment stage includes memory or other cognitive complaints. Dementia, the last stage, results in loss of memory, disorientation, increased anxiety, or aggregation. The FDA approved medications are cholinesterase inhibitors and glutamate modulators.$^{4}$ These medications provide only symptomatic relief. Medicinal plants can delay the progression of the disease.

\section{Natural products against AD}

Recently natural products have gained importance because of fewer side effects as compared to synthetic products. Plants that have shown activity against Alzheimer's are Ginkgo biloba, Withania somnifera, Huperzia serrata, Moringa olifera, Bacopa monnieri, Uncaria rhynchophylla, Galanthus woronowii, Curcuma longa, Panax ginseng, Centella asiatica, Glycyrrhiza glabra, Rosmarinus officinalis, etc.

\section{Ginkgo biloba}

EGb 761 is a standardized extract of Ginkgo biloba leaves which contains approximately $24 \%$ flavone glycosides and $6 \%$ terpene lactones. The flavone glycosides consist of quercetin, kaempferol, and isorhamnetin and terpene lactones consist of $\mathrm{A}, \mathrm{B}$, and $\mathrm{C}$ ginkgolides and bilobalide. In Germany, EGb 761 is approved for the treatment of dementia. It enhances SOD, catalase, and glutathione peroxidase (GPX) activities. ${ }^{5}$ EGb 761 offers protection against $A \beta$ induced neurotoxicity by preventing neuronal apoptosis and by blocking ROS accumulation, glucose uptake, mitochondrial dysfunction, and activation of ERK and JNK pathways. ${ }^{6}$ Free circulating cholesterol can also affect APP processing and amyloidogenesis. Yao et al. ${ }^{7}$ have shown that the levels of circulating free cholesterol were lowered and that the production of brain $\beta$ APP and $A \beta$ peptides were inhibited in EGb 761 treated older rats.

\section{Glycyrrhiza glabra}

Glycyrrhiza glabra belongs to Fabaceae family and includes many compounds like saponin, flavonoids, isoflavonoids, stilbenoids, and coumarins. Glycyrrhizin is the bioactive molecule present in it. It inhibits ROS generation, cytotoxicity, and GSH downregulation. An aqueous extract of Glycyrrhiza glabra when administered in 1-monthold rats orally for 6 weeks improved memory and learning. ${ }^{8}$ Hence it can be helpful in AD.

\section{Moringa olifera (MO)}

This plant belongs to the Morinngaceace family. Bioactive compounds like alkaloids, tannins, flavonoids, isothiocyanates, and saponin are present in MO. It causes an increase in SOD and catalase and a reduction in lipid peroxides (LPO) levels. This antioxidant activity might be the reason for improved cognition. ${ }^{9}$ It restores the disturbed brain monoamines levels, enhances memory, and provides protection from neurodegeneration. MO also decreases tau hyperphosphorylation in hyperhomocysteinemia rats. ${ }^{10}$

\section{Bacopa monniera (Brahmi)}

It is classified into Scrophulariaceae family and is found mostly in India and other Asian countries. It is used as a nootropic agent. Alkaloids and saponin are the chemical compounds present in Brahmi. Bacoside $\mathrm{A}$ is the active molecule in Brahmi. It prevents $\mathrm{A} \beta$ aggregation and formation of fibrils, prevents the reduction in SOD activity and decrease the LPO levels. ${ }^{11}$ 


\section{Centella asiatica}

Centella asiatica belongs to the Umbelliferae family. It is mainly found in India, Sri Lanka, and Bangladesh. It contains asiatic acid, asiaticosides, triterpenes, sapogenins, glycosides, madecassic acid, etc. It shows antioxidant effect by causing an increase in SOD, GPX (glutathione peroxidase), and catalase enzymes. ${ }^{12}$

\section{Panax ginseng}

It belongs to the Araliaceae family and contains triterpenoid dammarane glycosides. It inhibits $\beta$ - and $\gamma$-secretase activity, activates the nonamyloidogenic pathway, inhibits acetylcholinesterase activity and $A \beta$-induced neurotoxicity, and decreases $A \beta$-induced production of reactive oxygen species and neuroinflammatory reactions..$^{13}$ Panax ginseng was administered for 12 weeks in an open label study. To monitor the cognitive performance, Mini-mental state examination (MMSE) and Alzheimer's disease assessment scale (ADAS) score were used. In this study, it was observed that supplementation of Panax ginseng powder improved cognition in $\mathrm{AD}$ patients. ${ }^{14}$

\section{Rosmarinus officinalis}

It belongs to the Lamiaceae family and possesses antioxidant properties. Carnosol and carnosic acid are the potent antioxidants present in rosemary leaves. Carnosoic acid is involved in the synthesis of nerve growth factor (NGF). NGF is necessary for the growth and maintenance of nerve tissue. It maintains the levels of GPX and SOD activity. ${ }^{15}$

\section{Galanthus woronowii}

It belongs to the Amaryllidaceae family and consists of Galanthamine as the active compound. Galanthamine is a selective, reversible and competitive $\mathrm{AChE}$ inhibitor. It has shown improvement in memory and cognition in $\mathrm{AD}$ subjects. Currently, Galanthamine is the most widely approved AChE inhibitor approved by Food and Drug Administration (FDA) against AD. ${ }^{16}$

\section{Uncaria rhynchophylla}

It is a chinese herbal drug that belongs to the Rubiaceae family. Aqueous extract of Uncaria rhynchophylla showed a significant reduction in A $\beta$ fibril formation. Oxindole and indole alkaloids present in Uncaria rhynchophylla might be responsible for this effect. ${ }^{17}$

\section{Huperzia serrata}

It belongs to the Lycopodiaceae family and is widely distributed as a nootropic agent. Huperzine A is the active constituent and an AChE inhibitor used in Schizophrenia. To develop neuronal ischemia, oxygen glucose deprivation (OGD) was induced in PC12 cells. This leads to cell death and increased lipid peroxidation and SOD levels. Cells treated with Huperzine A showed reduced oxidative damage and increased survival. Glutamate-induced neurotoxicity was also attenuated by Huperzine A. ${ }^{18}$ These studies show the potential of Huperzia serrata in the treatment of $\mathrm{AD}$.

\section{Withania somnifera (Ashwagandha)}

It is a neuroprotective plant belonging to the Solanaceae family It is used as a nerve tonic in Ayurveda. It has calming properties and reduces stress. In $A \beta$ (25-35) induced rats, oral administration of Withanoside IV attenuated axonal, dendritic, and synaptic loss and memory deficits. Enhancement of cholinergic activity by increasing the acetylcholine content and cholineacetyl transferase activity was seen in rats after administration of aqueous extract of $W$. somnifera .${ }^{19}$ In mice, methanol extract of $W$. somnifera reversed the amyloid induced memory defecit. ${ }^{20}$

\section{Curcuma longa (Curcumin)}

It is classified into Zingiberaceae family. It has antioxidant, antiinflammatory, and anti-amyloidogenic properties and hence it has the potential to be used in $\mathrm{AD}$ treatment. In vitro and in vivo studies show that curcumin prevents $\mathrm{A} \beta$ aggregation and the formation of fibrils. There was a reduction in oxidative stress, inflammation, and cognitive deficits when $A \beta$ treated rats were given curcumin. ${ }^{21}$ In Tg2576 mice model of $\mathrm{AD}$, the antioxidative and anti-inflammatory potential of curcumin is reported. The anti-inflammatory effect occurs due to inhibition of NF-kB induced iNOS, COX (cyclooxygenase)-2, and inflammatory cytokine Production. ${ }^{22}$

\section{Conclusion}

Natural products are gaining popularity nowadays. Synthetic drugs show some serious side effects. This can be overcome by natural drugs that have low or no side effects. Medicinal plants can improve the quality of life of AD patients. Mechanism of actions of many medicinal plants is still not clear. Hence, trials involving larger populations are required to fully understand their role in Alzheimer's disease.

\section{Acknowledgments}

None.

\section{Conflicts of interest}

Declare if any conflict of interest exists.

\section{Funding}

None.

\section{References}

1. Harman D. Alzheimer's disease A hypothesis on pathogenesis. $J \mathrm{Am}$ Aging Assoc. 2000;23(3)147-161.

2. Wang R, Reddy PH. Role of Glutamate and NMDA Receptors in Alzheimer's Disease. J Alzheimer's Dis. 2017;57(4)1041-1048.

3. Alzheimer's Association. 2009 Alzheimer's disease facts and figures. Alzheimer's Dement. 2009;5(3)234-270.

4. FDA-approved treatments for Alzheimer's. 2019.

5. Chen L, Zhang C, Han Y, et al. Gingko biloba Extract (EGb) Inhibits Oxidative Stress in Neuro 2A Cells Overexpressing APPsw. Biomed Res Int. 2019;20191-10199.

6. Essa MM, Vijayan RK, Castellano-Gonzalez G, et al. Neuroprotective Effect of Natural Products Against Alzheimer's Disease. Neurochem Res. 2012 Sep 22;37(9)1829-842.

7. Yao Z-X, Han Z, Drieu K, Papadopoulos V. Ginkgo biloba extract (Egb 761 ) inhibits $\beta$-amyloid production by lowering free cholesterol levels. $J$ Nutr Biochem. 2004;15(12):749-756.

8. Chakravarthi KK, Avadhani R. Beneficial effect of aqueous root extract of Glycyrrhiza glabra on learning and memory using different behavioral models An experimental study. J Nat Sci Biol Med. 2013;4(2):420-425.

9. Ganguly R. Effect of Moringa Oleifera in Experimental Model of Alzheimer's Disease Role of Antioxidants. Ann Neurosci. 2005;12(3):3336 
10. Mahaman YAR, Huang F, Wu M, et al. Moringa Oleifera Alleviates Homocysteine-Induced Alzheimer's Disease-Like Pathology and Cognitive Impairments. J Alzheimer 's Dis. 2018;63(3):1141-1159.

11. Abdul Manap AS, Vijayabalan S, Madhavan P, et al. Bacopa monnieri, a Neuroprotective Lead in Alzheimer Disease A Review on Its Properties, Mechanisms of Action, and Preclinical and Clinical Studies. Drug Target Insights. 2019;13117739281986641.

12. Chen CL, Tsai WH, Chen CJ, et al. Centella asiatica extract protects against amyloid $\beta 1-40$-induced neurotoxicity in neuronal cells by activating the antioxidative defence system. J Tradit Complement Med. 2016;6(4):362-369.

13. Kim HJ, Jung SW, Kim SY, et al. Panax ginseng as an adjuvant treatment for Alzheimer's disease. J Ginseng Res. 2018;42(4):401-411.

14. Lee ST, Chu K, Sim JY, et al. Panax Ginseng Enhances Cognitive Performance in Alzheimer Disease. Alzheimer Dis Assoc Disord. 2008;22(3):222-226.

15. Wijeratne SSK, Cuppett SL. Potential of rosemary (Rosemarinus officinalis L) Diterpenes in preventing lipid hydroperoxide-mediated oxidative stress in Caco-2 cells. J Agric Food Chem. 2007;55(4):11931199.

16. Marco L, Carreiras M. Galanthamine, a Natural Product for the Treatment of Alzheimers Disease. Recent Pat CNS Drug Discov. 2006;1(1):105-111.
17. Fujiwara H, Iwasaki K, Furukawa K, Seki T, He M, Maruyama M, et al. Uncaria rhynchophylla, a Chinese medicinal herb, has potent antiaggregation effects on Alzheimer's $\beta$-amyloid proteins. $J$ Neurosci Res. 2006;84(2):427-433.

18. Wang R, Xi CT. Neuroprotective effects of huperzine A A natural cholinesterase inhibitor for the treatment of Alzheimer's disease. Neurosignals. 2005;14(1-2):71-82.

19. Schliebs R, Liebmann A, Bhattacharya S, et al. Systemic administration of defined extracts from Withania somnifera (Indian ginseng) and Shilajit differentially affects cholinergic but not glutamatergic and GABAergic markers in rat brain. Neurochem Int. 1997;30(2)181-190.

20. Kuboyama T, Tohda C, Komatsu K. Neuritic regeneration and synaptic reconstruction induced by withanolide A. Br J Pharmacol. 2005;144(7):961-971.

21. Cole GM, Teter B, Frautschy SA. Neuroprotective effects of curcumin. Adv Exp Med Biol. 2007;595:197-212.

22. Ono K, Hasegawa K, Naiki H, et al. Curcumin Has Potent AntiAmyloidogenic Effects for Alzheimer's $\beta$-Amyloid Fibrils In Vitro. $J$ Neurosci Res. 2004;75(6):742-750. 